\title{
Developing a children's glaucoma passport: mapping innovations in healthcare
}

Eye (2017) 31, 1635-1638; doi:10.1038/eye.2017.115; published online 16 June 2017

Living with childhood glaucoma is an enormous challenge. Involving children and their carers on this journey helps to maximize self-care, optimize quality of life, and gives children and parents a voice in the management of this potentially devastating disease.

Patient self-management tools in healthcare can improve effectiveness and efficiency of healthcare delivery and are increasingly used in the care of chronic disease. ${ }^{1}$ A widely reported example is the adult asthma passport; now broadly implemented and available for children. ${ }^{2,3}$ Children's passports are also available for epilepsy and chronic skin conditions. This is the first description in the world literature of a Children's Glaucoma Passport.

The successful adoption of a new innovation, such as a patient passport by a clinician or healthcare system is multifaceted. One of the better-known theoretical approaches to this is Rogers' theory on diffusion of innovation. ${ }^{4}$ This postulates that there are five factors influencing adoption or diffusion of a new clinical behavior, which are as follows: (1) relative advantage, (2) compatibility, (3) complexity, (4) trial-ability, and (5) observability. These five factors have previously been incorporated into a concept map following a systematic review by Greenhalgh et $a l^{5}$ in 2004; however, no concept model exists mapping innovation and implementation of a self-management tool in a pediatric context.

We developed and implemented a Children's Glaucoma Passport. Following this service improvement project, we used Rogers' theory to help us to map out a process of development, implementation, improvement and adoption (Figure 1). Initially, key drivers for passport creation were identified. Examples included complex treatment regimens delivered in many settings, patients as sub-optimal self-managers and communication gaps.

The passport was produced in consultation with parents and children. We asked parent and patient representatives for their guidance at the Second National Glaucoma Think-Tank (run in partnership with the International Glaucoma Association) a patient-public involvement event (PPI) reported previously. ${ }^{6}$ The data collected from this event was recorded, transcribed and analyzed using qualitative research methodology techniques. ${ }^{7}$ Informed consent was gained from all participants. The study adhered to the tenets of the Declaration of Helsinki and ethical approval was gained from the National Ethics Research Service (11/SW /0289).

Parents wanted a tool that effectively recorded the details of treatment plans, including drug/ drop regimens, refractive error, and glasses prescription and amblyopia (lazy eye) treatment plans. Other priorities suggested by parents were the importance of portability (A5 was agreed size) and a picture guide for correct drop administration. In common with the patientrelated outcome and experience measures, patient representatives emphasized the importance of both documents being carefully constructed to be quick to use with clearly presented information. ${ }^{6}$

In outline, the A5 sized passport (Figures 2a-c) contains the following sections:

1. 'Family and school' in which children can draw pictures of themselves, their family and school or stick pictures (Figure 2b).

2. Emergency contact numbers, names and contact numbers of care team, including eye surgeon, optometrist, orthoptist, secretary.

3. Child's medical history (systemic and ophthalmic). 


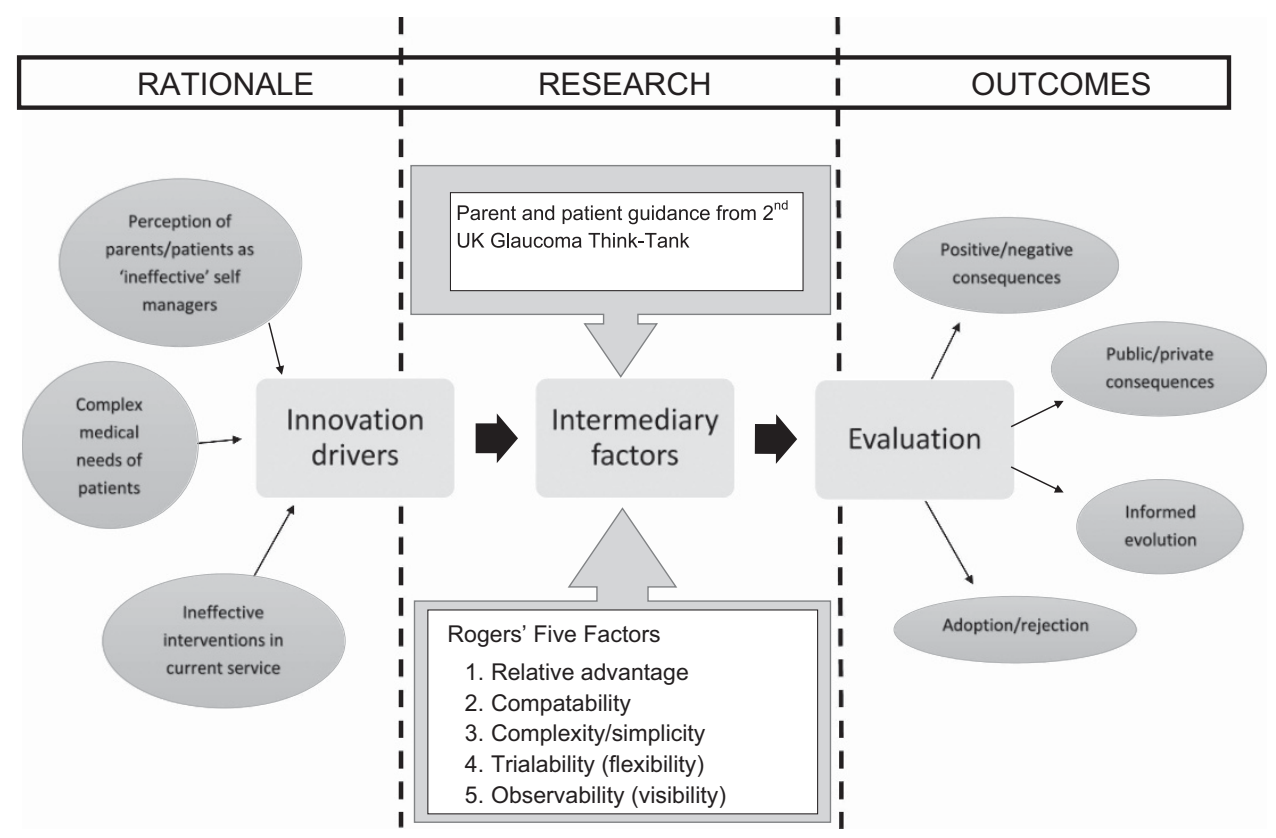

Figure 1 Concept model for mapping delivery of healthcare innovations.
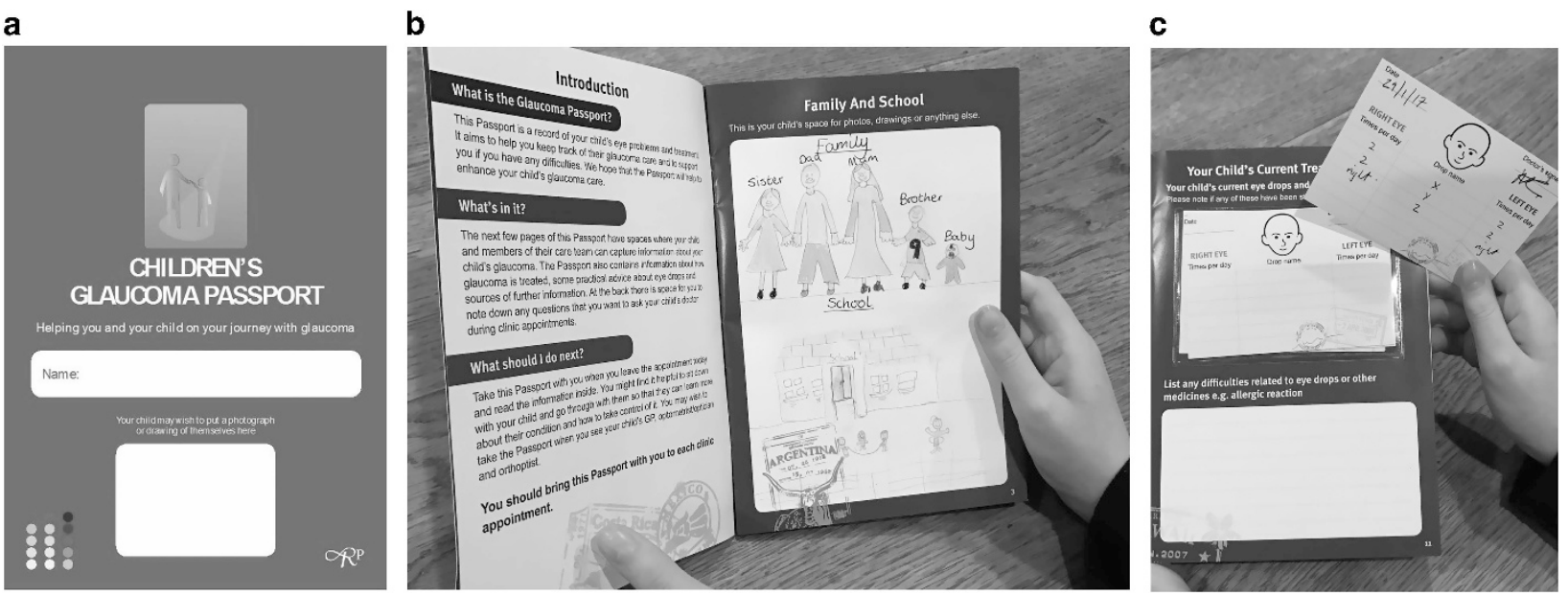

Figure 2 (a) Passport cover. (b) Passport pages 2-3. (c) Page 11 from passport; interchangeable drop treatment cards.

4. Systemic and ophthalmic treatment regime by means of interchangeable cards in wallet (Figure 2c).

5. A drops guide; how to put eye drops in correctly.

6. Frequently asked questions and answers.

7. Important things to remember for next appointment; a 'Parents' Survival Guide'.

8. Approved sources of further information.

After initial development of the Children's Glaucoma Passport, the process of implementation into clinical practice was evaluated. Feedback from parents and children was analyzed over a 12-month period. The data were also collected from over 20 stake-holders; parents, ophthalmologists, allied health professionals, and charitable fund-holders. The data were collected using questionnaires, and recorded telephone interviews. These suggested that we had satisfied Roger's theory with examples as follows:

'It prevents patients/parents from losing bits of written information; as it is all in one known place... vision, patching, treatment'

Trainee ophthalmologist, Birmingham 
'Mum uses it for everything, for all appointments, GP, hospital...'

Mother of a 3-year-old son, with little spoken English

'... the parents will write it in there ... if they haven't had three drops a day as prescribed, only two ... they'll explain why..'

\section{Orthoptist}

'[the surgeon] uses the medication cards to explain to them what to have... they've used the passport to explain to the other children and the teacher has actually used ..... to understand what she's doing.'

\section{Mother with 5-year-old son}

'the passport improves safety, efficiency, communication and therefore outcomes.'

\section{Ophthalmologist}

Parents told us that the most useful section of the passport was the interchangeable cards for the treatment regime (Figure 2).

All parents said that the passport 'made things easier at home' and 'made easier in the clinic'; however, only a third said that children themselves engaged with using the passport.

Our reflections are, after implementing the Children's Glaucoma Passport, are as follows:

1. Parents seem pleased when they receive the passport. The passport indicates that their child's glaucoma, is taken sufficiently seriously that the disease justifies a specifically developed document. Parents respond extremely positively to this.

2. This importance of glaucoma management is better communicated to all of those involved in the child's care; from teachers, opticians, relatives, carers, babysitters, pediatricians, and others. Parents say that this makes their life easier.

3. At the point, the passport is filled in and handed over by the doctor, this moment is a useful point of the consultation; a physical representation of transfer of primary responsibility for medical management from the ophthalmology team to the family, until the next point of contact.

4. Time efficiency is improved especially regarding verifying past treatment regimes. Colleagues sometimes consider they do not have enough time to use this new document in their practice. Our experience is to the contrary; this document saves time.

5. Motivation and adherence to treatment is improved.
6. Errors and miscommunication regarding drop/medication regimes are reduced.

7. Patient/parent education is improved, comprehensive and documented.

8. Clearly presented and documented information for families helps protect doctors medicolegally and children from harm.

9. Children receive care in disparate contexts, across a variety of healthcare, educational and geographical boundaries. The passport travels across these boundaries and closes these communication gaps.

10. Often, early in a consultation, parents open the passport and hand over the card from the treatment wallet (Figure 2c) reflecting the importance placed on this.

11. Parents' ability to recognize an emergency situation is improved and gives them clear emergency contact numbers.

We learned from our qualitative analysis that we could improve younger childrens' engagement with the passport. We plan to incorporate sticker charts and more child-friendly illustrations such as cartoons, for our younger users in future iterations.

There are advantages in having versions for younger children and a different version for teenagers though this would have cost implications.

Our concept model, presented here, has broad application for the development of all healthcare innovations and their implementation.

Having developed, implemented and evaluated the Children's Glaucoma Passport, it has now become the standard of care for all children with glaucoma coming through our unit.

\section{Conflict of interest}

The authors declare no conflict of interest.

\section{Acknowledgements}

We thank Birmingham Children's Hospital Charities for generously supporting this project.

\section{References}

1 Holman H, Lorig K. Patient self-management: a key to effectiveness and efficiency in care of chronic disease. Public Health Rep 2004; 119: 239-243.

2 Newell K, Basi T, Hume S. Development of a patient passport in asthma management. Nurs Stand 2014; 29: 37-42.

3 Roger L, Zemek MD, Sanjit KG, Francine MD. Systematic review of randomized controlled trials examining written action plans in children: what is the plan? Arch Pediatr Adolesc Med 2008; 162: 157-163.

4 Sanson-Fisher RW. Diffusion of innovation theory for clinical change. Med J Aust 2004; 180: 55. 
5 Greenhalgh T, Robert G, Macfarlane F, Bate P, Kyriakidou O. Diffusion of innovations in service organizations: systematic review and recommendations. Milbank $Q$ 2004; 82: 581-629.

6 Somner JEA, Sii F, Bourne RR, Cross V, Burr JM, Shah P. Moving from PROMs to POEMs for glaucoma care: a qualitative scoping exercise. Invest Ophthalmol Vis Sci 2012; 53 5940-5947.

7 Braun V, Clarke V. Using thematic analysis in psychology. Qual Res Psychol 2006; 3: 77-101.

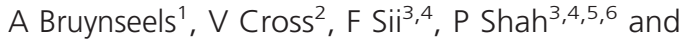$$
\text { J Abbott }{ }^{4,7}
$$

${ }^{1}$ Frimley Park Hospital, Camberley, UK

${ }^{2}$ Institute of Health, Faculty of Education, Health and

Wellbeing, University of Wolverhampton,

Wolverhampton, UK

${ }^{3}$ Department of Ophthalmology, Queen Elizabeth

Hospital Birmingham, University Hospitals

Birmingham NHS Foundation Trust, Birmingham, UK
${ }^{4}$ Birmingham Institute for Glaucoma Research, Institute for Translational Medicine, University Hospitals Birmingham NHS Foundation Trust, Birmingham, UK

${ }^{5}$ Centre of Health and Social Care Improvement,

Faculty of Education, Health and Wellbeing, University of Wolverhampton, Wolverhampton, UK

${ }^{6}$ University College London, London, UK

${ }^{7}$ Birmingham Children's Hospital, Birmingham, UK

Study material presented at the Royal College of Ophthalmologists, Annual Congress 2016.

Correspondence: J Abbott, Birmingham Children's Hospital, Steelhouse Lane, Birmingham, B4 6NH, UK

Tel: +44 (0)121333 9465;

Fax: +44 (0)121 3339461 E-mail: Joseph.abbott@bch.nhs.uk 